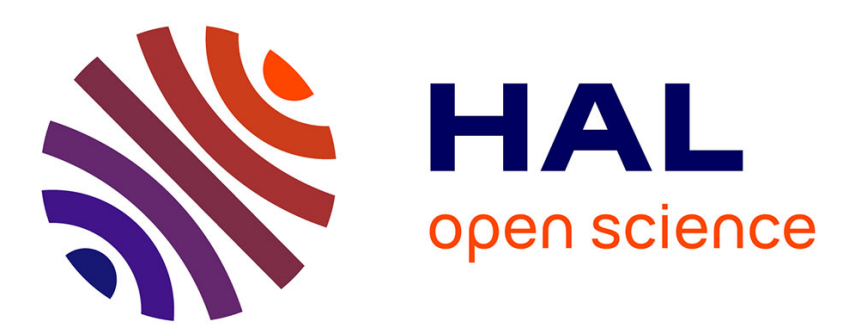

\title{
La fiscalité des transports en France. Du développement de l'offre-la gestion de la demande
}

\author{
Emile Quinet
}

\section{To cite this version:}

Emile Quinet. La fiscalité des transports en France. Du développement de l'offre-la gestion de la demande: Du développement de l'offre à la gestion de la demande. 2011, pp.5-29. 10.1051/futur/3705 . halshs-00754565

\section{HAL Id: halshs-00754565 https://hal-pjse.archives-ouvertes.fr/halshs-00754565}

Submitted on 29 May 2020

HAL is a multi-disciplinary open access archive for the deposit and dissemination of scientific research documents, whether they are published or not. The documents may come from teaching and research institutions in France or abroad, or from public or private research centers.
L'archive ouverte pluridisciplinaire HAL, est destinée au dépôt et à la diffusion de documents scientifiques de niveau recherche, publiés ou non, émanant des établissements d'enseignement et de recherche français ou étrangers, des laboratoires publics ou privés. 


\section{La fiscalité des transports en France}

DU DÉVELOPPEMENT DE L'OFFre

À LA GESTION DE LA DEMANDE

PAR ÉMILE QuineT ${ }^{I}$

Les débats vont bon train, en France, depuis plusieurs mois, s'agissant de la pertinence et de l'efficacité de la politique fiscale nationale. Mais il est un secteur relativement épargné par ces discussions, nous dit ici Émile Quinet : celui des transports dont les taxes, pourtant assez nombreuses, sont finalement peu discutées. Pourtant, il $y$ aurait matière à réflexion, comme il le montre ici de manière très détaillée.

En effet, la taxation des transports en France vise aujourd'hui essentiellement à fournir des ressources financières pour adapter l'offre de transports à la demande des usagers, alors que d'autres fonctions telles la recherche de l'équité, mais aussi et surtout l'orientation de la demande, par exemple pour lutter contre les externalités (pollution, accidents, congestion...), pourraient (sinon devraient) prévaloir. Après un état des lieux très complet de la fiscalité des transports, Émile Quinet en montre bien le caractère très financier ; il souligne aussi le manque d'efficacité de la tarification des transports, avant de se pencher sur les gains possibles d'une bonne orientation de la demande au moyen d'instruments fiscaux. Bien entendu, il reste beaucoup d'obstacles, tant en termes d'équité, d'acceptabilité sociale, de corporatisme qu'en matière administrative ; mais la situation pourrait changer sous l'influence de divers facteurs tels que les nouveaux moyens technologiques en matière d'information et de communication, les expériences menées à l'étranger et relayées par I'Union européenne, ou encore le climat favorable aux actions proenvironnementales né du Grenelle de l'environnement.

I. Membre de l'Académie des technologies ; chercheur PSE-ENPC (Paris School of Economics, École nationale des ponts et chaussées). 
Cet article montre qu'il existe, une fois n'est pas coutume, dans le secteur des transports, de réelles marges de manœuvre pour orienter la demande et développer des politiques en phase avec les exigences notamment environnementales de notre époque. II serait dommage de ne pas en profiter ou de le faire sans un certain effort pédagogique à l'égard de l'opinion. S.D.

$\mathbf{L}$ es transports constituent un domaine privilégié pour les interventions financières de la puissance publique. Les taxes et subventions qui touchent l'usager, soit directement soit indirectement par l'intermédiaire des opérateurs, y représentent des montants particulièrement élevés. En France, les comptables nationaux les évaluent à 70 milliards d'euros, ce qui équivaut en gros au quart de la dépense nationale de transport ou encore à 3,5\% du produit intérieur brut (PIB). Sur ces 70 milliards d'euros, près de 40 milliards correspondent à des taxes spécifiques en sus des taxes de droit commun - TVA (taxe sur la valeur ajoutée) au taux normal, impôts sur les sociétés... - qui frappent les transports au même titre que les autres secteurs. En sens inverse, les pouvoirs publics dépensent environ 45 milliards d'euros dans les transports, dont to milliards d'euros sous forme de subventions aux opérateurs et 35 milliards d'euros en dépenses directes.

Ces taxes sont multiples. Chaque automobiliste sait bien que le prix du carburant est essentiellement composé de taxes. Mais qui connaît la taxe sur les contrats d'assurance automobile ou encore les redevances domaniales payées par les sociétés d'autoroutes à péage — et donc indirectement par leurs clients ? Qui sait que les transports publics urbains sont subventionnés dans une proportion variable selon les villes mais qui ne descend jamais en dessous de la moitié de leur coût?

À une époque où l'on s'interroge sur l'utilisation de l'argent public et où de nombreuses voix s'élèvent pour une remise à plat de la fiscalité, il n'est pas inutile d'examiner la cohérence et la logique des échanges financiers dont les montants viennent d'être évoqués, en centrant l'examen sur les taxes et les subventions spécifiques. On les analysera au regard des trois critères classiques : les montants des ressources récoltées, l'équité de la mesure et ses effets en termes d'incitation à l'efficacité économique. On retrouve ces trois types d'arguments aussi bien dans les discours spécialisés des économistes que dans les débats de l'opinion publique. Ainsi, pour la taxe carbone, on s'est interrogé sur l'ampleur des recettes qu'elle engendrerait, sur sa capacité à réduire les émissions de gaz à effet de serre et sur son caractère plus ou moins équitable en fonction de ses modalités de mise en œuvre. De même, les débats récurrents sur l'ISF (impôt de solidarité sur la fortune) mettent en avant le faible montant que rapporte cet impôt, ses conséquences en matière de redistribution des richesses, et son effet négatif sur les mouvements de capitaux. Nombre de taxes apparaissent ainsi de façon récurrente dans le débat public, comme par exemple la TVA et son effet négatif sur la consommation, donc sur la croissance, ou encore les taxes et charges 
sociales qui s'appliquent aux salaires et dont on met en exergue les effets néfastes sur l'emploi.

Mais curieusement, les taxes frappant les transports soulèvent peu de discussion, si ce n'est pour estimer qu'elles sont trop élevées, une position bien naturelle de la part de ceux qui les paient, c'est-à-dire nous tous. Pourtant, il y a beaucoup à dire à leur sujet, comme voudrait le faire apparaître l'analyse qui suit, d'où il ressort qu'on les a instaurées en fonction de leur rendement, pour fournir des moyens financiers permettant de répondre aux besoins qui se manifestent, à la demande et sans trop choquer les soucis d'équité. Mais on n'a pas - pas assez — tenu compte de ce que les besoins qui s'expriment naturellement ne sont pas tous socialement bénéfiques ; certains d'entre eux engendrent des coûts collectifs importants et il vaut mieux alors les réduire plutôt que les satisfaire intégralement. On n'a pas assez utilisé la taxation pour orienter la demande afin d'améliorer l'efficacité.

On pourrait cependant beaucoup gagner sur ce dernier point, par un système de taxes rapportant les mêmes sommes, voire plus, mais mieux ciblées en termes d'orientation de la demande. Avant de développer ces aspects, il faut d'abord expliciter davantage les trois effets de la taxation précédemment cités, et dresser le tableau des taxes et subventions frappant les transports.

\section{Les trois fonctions de la taxation}

Les taxes et subventions publiques ont trois grandes catégories de conséquences : elles fournissent des ressources financières aux autorités publiques, elles modifient la répartition des revenus et agissent sur l'équité de la société, elles modifient les choix des usagers.

Fournir des ressources pour financer les dépenses publiques, c'est la raison d'être initiale et toujours la destination finale des taxes, qu'elles servent au financement du budget général ou qu'elles soient affectées à des catégories particulières de dépenses publiques, qu'elles frappent indistinctement toutes les activités ou qu'elles soient spécifiques à un secteur particulier, en l'occurrence les transports.

Le deuxième effet des interventions financières publiques, c'est leur conséquence sur l'équité et la distribution des richesses : dans quelle mesure la taxe ou la subvention en cause touche-t-elle telle ou telle catégorie de la population ? Contribue-t-elle à réduire (effet dit progressif) ou à augmenter (effet dit régressif) l'inégalité dans la répartition des revenus?

Le troisième effet est d'ordre incitatif : dans la mesure où la taxe contribue à orienter les choix des usagers, elle agit sur l'efficacité de la machine économique. En augmentant ou réduisant la fiscalité qui s'applique à tel type de véhicule ou de déplacement, ou à tel mode, les pouvoirs publics ont la capacité d'en diminuer ou d'en développer l'usage, et d'influer sur la demande, tout comme une entreprise privée le fait en agissant sur les prix des produits qu'elle met sur le marché. Mais alors que l'entreprise privée joue 


\section{L'ORIENTATION DU CHOIX DES USAGERS} ET LES EFFETS EXTERNES

Les externalités ou effets externes se présentent dans les situations où un agent économique, le pollueur, occasionne une gêne ou un coût - ou procure un profit - à un autre agent, le pollué, sans que ce dernier puisse influer sur le niveau de la gêne ou du coût qu'il subit.

Un exemple en matière de transport est celui d'un avion qui occasionne des nuisances sonores aux riverains de l'aéroport. Par définition, en quelque sorte, les coûts externes sont la différence entre les coûts collectifs et les coûts privés. En présence de coûts ex- ternes, celui qui les provoque n'a aucune incitation à limiter la gêne qu'il cause, contrairement à ce à quoi conduirait l'intérêt général. On montre que la recherche de cet intérêt général mène, non pas à annuler la pollution (ce qui entraînerait des coûts excessifs), mais à la limiter au niveau où le coût de dépollution égale le coût de la gêne occasionnée pour le pollué. II s'agit en somme d'une version élaborée du principe pollueur-payeur : chacun doit payer la gêne qu'il occasionne aux autres.

É.Q.

de cet instrument pour maximiser son profit, les pouvoirs publics peuvent - ou tout au moins doivent - l'utiliser dans un autre but, celui de combler l'écart entre le coût privé du déplacement et son coût collectif, ou, en d'autres termes, d'internaliser les coûts externes, ces coûts engendrés par les situations où un agent économique, le pollueur, occasionne une gêne ou un coût - ou procure un profit - à un autre agent, le pollué, sans que ce dernier puisse influer sur le niveau de la gêne ou du coût qu'il subit.

L'analyse économique enseigne à ce sujet un certain nombre de résultats fondamentaux, rappelés dans l'encadré ci-dessus, qui reviennent à l'application du principe pollueur-payeur, et dont l'essence est que, pour une orientation optimale des décisions des usagers du point de vue de la collectivité, il convient que chaque agent générateur d'un effet externe paie le coût ainsi occasionné aux autres, pour lui faire prendre conscience de la gêne qu'il engendre, non pour punir le fauteur par une sorte de loi du talion, mais pour l'inciter à réduire cette gêne.

Ce principe constitue la doctrine de l'Union européenne. L'encadré page ci-contre fournit quelques rappels concernant l'évaluation des coûts externes, nombreux et particulièrement importants dans les transports : la pollution de l'air, le bruit, l'effet de serre, la congestion et la sécurité, les atteintes au paysage et aux milieux ambiants. Il met en évidence leurs importances relatives et fournit des estimations des coûts externes principaux pour chacun des modes de transport, c'est-à-dire des taxes qu'il conviendrait d'appliquer dans une perspective pollueur-payeur d'orientation de la demande. Retenons-en quelques conclusions générales :

- Parmi les modes de transport, c'est la route qui est la principale source de coûts externes. 
- Les coûts externes les plus élevés sont ceux issus de la congestion et de la sécurité ; les coûts d'environnement viennent bien après.

- L'effet de serre présente un coût limité, sans rapport avec l'importance des débats auxquels donne lieu le réchauffement planétaire.

- Les effets externes varient considérablement selon le type de véhicule, le lieu et l'heure du trajet ; ce point est particulièrement marqué pour la congestion.

Notons enfin qu'à ces effets externes s'ajoutent d'autres coûts éventuels non naturellement payés par les usagers et qu'il est nécessaire d'internaliser dans une perspective d'orientation de la demande : il s'agit des coûts exposés par la puissance publique dans la gestion des infrastructures dont l'usage est libre ; c'est le cas pour les routes ordinaires et les autoroutes sans péage dont l'usage est gratuit alors que leur construction et leur entretien occasionnent des coûts collectifs à la charge des autorités publiques. Il est alors nécessaire, dans un but de bonne orientation des choix, de taxer les véhicules en fonction des coûts qu'ils engendrent.

Chaque taxe a un but principal énoncé dans les textes qui la fondent, ou qui ressort de la manière dont elle est gérée, ou que l'on peut apprécier par ses effets, et qui permet de la ranger prioritairement dans l'une des trois catégories ci-dessus (source de revenu, redistribution ou orientation des choix des usagers) ; mais toutes les taxes ont des effets sur chacune des trois fonctions. Ainsi, certaines taxes qui rapportent des sommes élevées, n’ont que peu de conséquences sur la répartition des richesses et n'influencent guère les choix des consommateurs ; pour prendre des exemples hors du secteur des transports, on peut considérer qu'en première analyse c'est le cas de la TVA. D'autres ont des conséquences essentiellement distributives, comme la taxation des plus-values mobilières. Pour d'autres, enfin, l'impact essentiel concerne les choix des consommateurs ; c'est le cas de la taxe sur les alcools.

\section{LES COOTS EXTERNES DANS LES TRANSPORTS}

Les coûts externes concernent d'abord - ce sont les plus connus de l'opinion publique - les atteintes à l'environnement : bruit, pollution de l'air et des sols, atteintes aux paysages, réchauffement planétaire, émission de déchets, morcellement des habitats fragmentés par les infrastructures linéaires, modification des possibilités de chalandise résultant de la traversée des agglomérations par les routes et les voies ferrées. En outre, la plupart des modes, et au premier rang la route, connaissent les phénomènes de congestion : la qualité du service exprimée en termes de temps de trajet ou de confort se dégrade quand le trafic augmente, chaque usager qui s'introduit sur une route - ou chaque avion qui s'insère dans le trafic aérien, ou chaque train que I'on veut insérer dans une grille horaire existante - occasionne une gêne à ceux qui y sont déjà. La sécurité est également un effet externe : chaque accident présente un coût collectif (soins, perte humaine, dégâts matériels) qui 


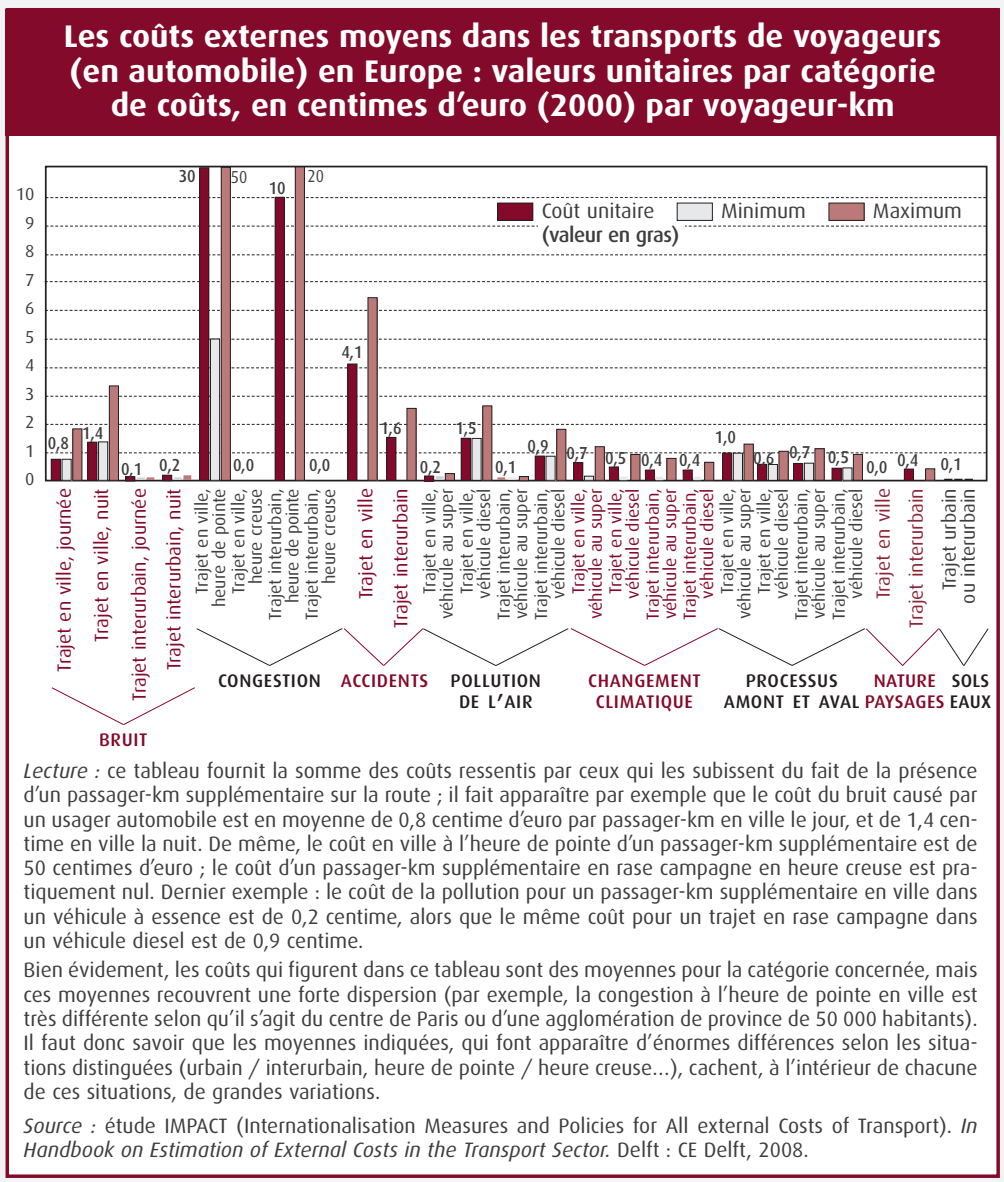

n'est pas supporté par ses protagonistes, et qui justifie l'intervention des pouvoirs publics et leurs actions pour en diminuer le nombre et les conséquences.

La plupart de ces effets impliquent des biens pour lesquels il n'existe pas de prix, car ce sont des biens non marchands : le temps, la vie humaine, l'air pur. Ils n'en ont pas moins une valeur économique et on peut évaluer cette valeur, c'est-à-dire les monétariser, en calculant les montants que ceux qui subissent ces effets externes seraient prêts à payer pour les éviter et les dommages qu'ils occasionnent. Les nombreuses études menées sur le sujet aboutissent à des valeurs convergentes, dont la synthèse la plus complète et la plus récente est fournie pour l'Europe par l'étude IMPACT ${ }^{1}$. On en extrait cidessus un graphique présentant les coûts externes moyens pour l'ensemble de l'Europe en matière de transport de voyageurs.

É.Q.

1. IMPACT (Internationalisation Measures and Policies for All external Costs of Transport). In Handbook on Estimation of External Costs in the Transport Sector. Delft : CE Delft, 2008. 


\section{Une fiscalité spécifique essentiellement financière}

\section{L'état des lieux}

Le classement des taxes spécifiques frappant les transports est présenté dans le tableau I, page suivante, où figurent les montants récoltés, l'assiette et les impacts sur chacune des trois catégories d'effets évoqués précédemment.

La majeure partie des quelque 38 milliards d'euros de taxes spécifiques provient de la route, et plus particulièrement de la taxation des carburants, qui représente plus de 24 milliards d'euros. Les taxes non routières s'élèvent à 8,4 milliards d'euros. On remarquera que l'on a classé dans les taxes spécifiques le «versement transport », qui ne frappe pas les utilisateurs de transports mais les entreprises situées dans les agglomérations et dont les employés bénéficient des transports urbains ; indirectement, c'est donc bien une taxation des transports. Le reste, soit 2,7 milliards d'euros, concerne le transport aérien. Le transport ferroviaire n'est pas soumis à taxation spécifique mais bénéficie de subventions - des taxes en négatif, en quelque sorte - ; c'est aussi le cas des transports collectifs urbains et régionaux, comme le montre le tableau 2, page suivante.

\section{L'accent mis sur le recouvrement de fonds}

Le tableau I montre que, des trois objectifs assignés à la tarification, le principal est la couverture des dépenses. C'est flagrant pour la taxation des carburants. La taxe intérieure sur les produits pétroliers (TIPP), créée juste après-guerre, avait et a toujours pour but d'augmenter les ressources publiques. La facilité de perception et l'acceptabilité de cet impôt ont fait son succès.

Quant aux taxes frappant l'aviation, elles sont majoritairement destinées à fournir des ressources aux aéroports et au service de la navigation aérienne ; liées au poids de l'avion, donc au nombre de passagers transportés, elles sont fondées essentiellement sur la capacité à payer ; elles n'ont pas, sauf la taxe sur les nuisances sonores, d'objectif de correction des effets externes ; ne dépendant pas des horaires, elles ne sont pas liées à la congestion des aéroports ni à la rareté des créneaux d'atterrissage et de décollage.

Le versement transport lui-même n'avait à l'origine, et n'a toujours d'autre but que de fournir des ressources aux collectivités locales pour le développement des transports collectifs. Il présente un certain caractère d'équité puisqu'il frappe les bénéficiaires des transports collectifs que sont les employeurs ; son effet incitatif pourrait être de dissuader les entreprises de s'installer dans les agglomérations, et donc indirectement de réduire les flux de transport dans l'agglomération, mais cet effet, pas forcément souhaitable d'ailleurs ${ }^{2}$, ne semble guère jouer.

2. Il faut plutôt inciter les entreprises à se grouper dans des agglomérations, c'est là que les synergies de voisinage ont le plus d'effet sur leur productivité. 


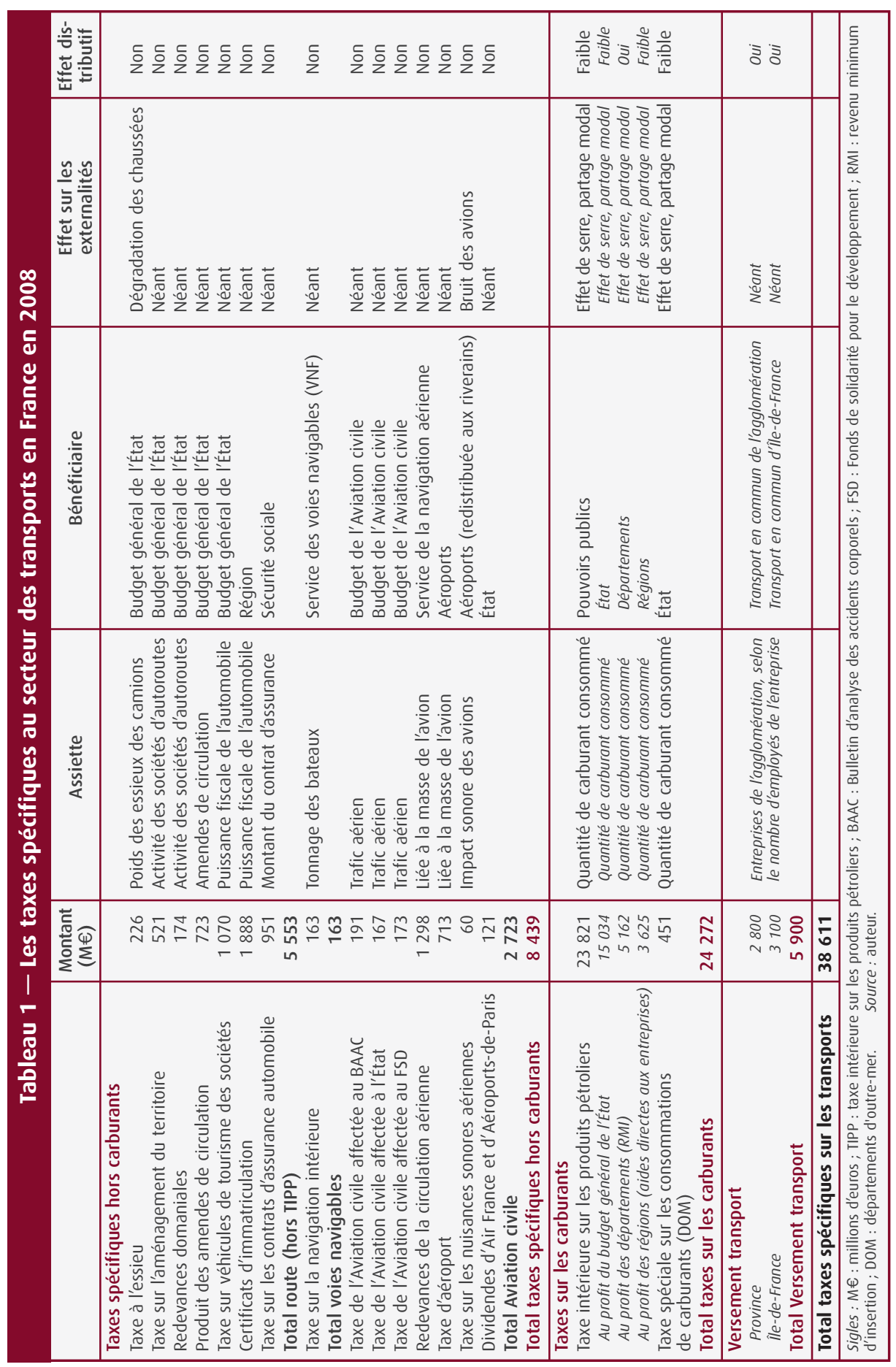




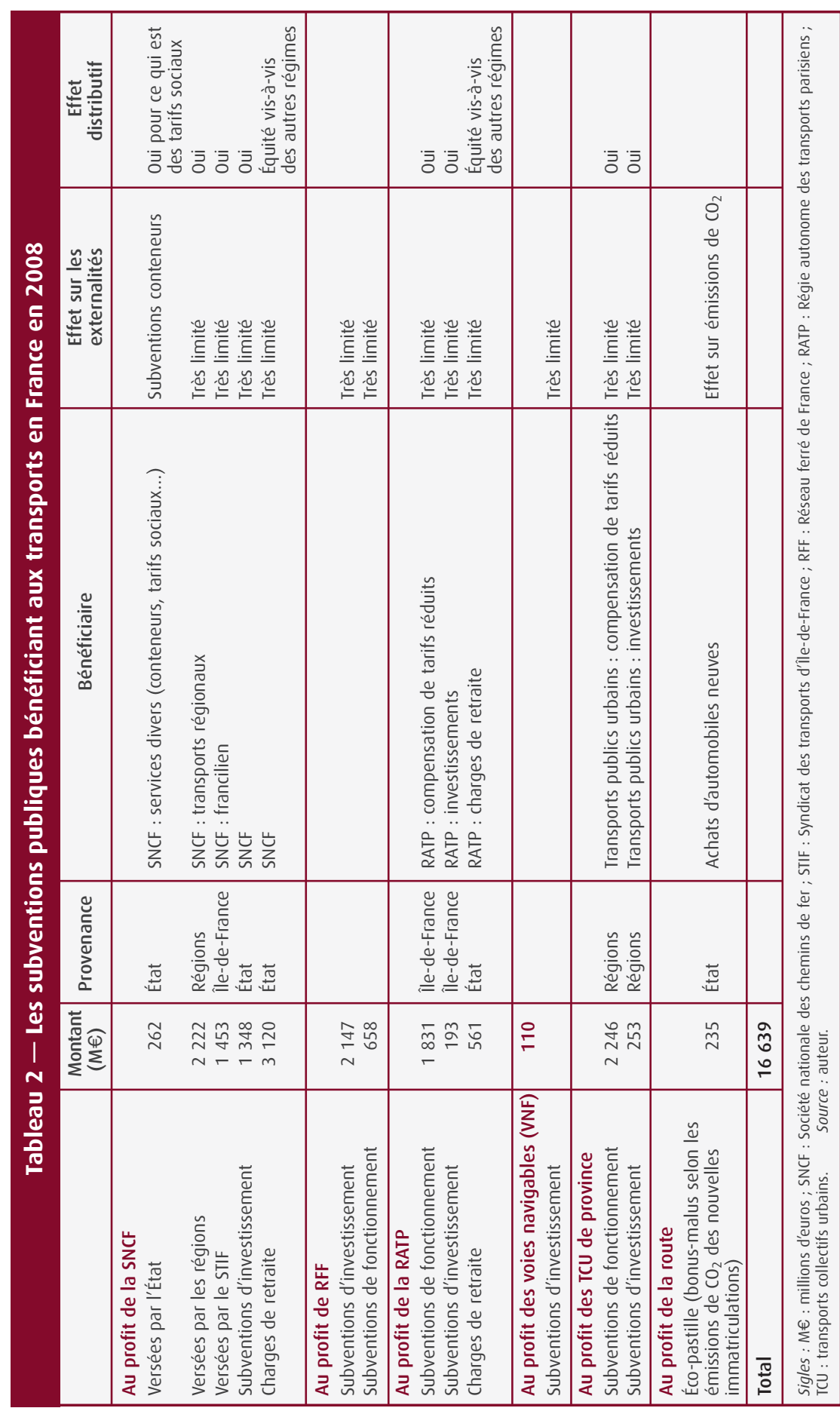


En outre, pour la plupart d'entre elles, c'est aux budgets généraux de l'État ou des collectivités territoriales qu'elles sont destinées, sans affectation prédéfinie. C'est le cas de toutes les taxes routières. Certes, pendant un temps, une liaison avait été établie pour le produit de la TIPP, mais cette liaison, qui a maintenant disparu, était de pure forme : l'État définissait séparément les montants financiers qu'il voulait consacrer à la route et les taux de la TIPP ; il en déduisait le pourcentage de la TIPP qui était affecté à la route, mais ce pourcentage non imposé a priori changeait au fil des ans, c'était le résultat de deux décisions séparées. Le ministère des Finances a en effet toujours veillé à une stricte application du principe de l'unicité budgétaire et a fait la chasse aux affectations spéciales qu'il a réussi, jusqu'à il y a quelques dizaines d'années, à vider de leur sens. Ultérieurement, le principe de l'unicité budgétaire s'est trouvé entamé, plusieurs services publics ont obtenu leur autonomie financière avec un budget propre et des recettes qui devaient équilibrer leurs dépenses. Mais cette affectation des taxes garde une ampleur limitée dans les transports ; on ne les trouve que pour les taxes d'aviation et les taxes portuaires, qui sont peu à peu devenues les ressources essentielles des gérants d'infrastructures des modes correspondants.

Les taxes spécifiques n'équilibrent cependant pas tout à fait les dépenses publiques en faveur du transport, qui s'élèvent à environ 45 milliards d'euros (voir encadré ci-contre), alors que les taxes spécifiques ne sont que de 38 milliards d'euros.

Ce déséquilibre d'ensemble cache d'ailleurs d'autres déséquilibres partiels. On voit (tableau I) que la route paie la plus grande part des taxes alors que les subventions vont majoritairement aux transports publics. Par ailleurs, l'État est le grand bénéficiaire des recettes alors que de volumineuses dépenses sont faites par les collectivités territoriales, comme le fait apparaître l'encadré ci-contre. Enfin, une analyse plus approfondie montrerait que le gros des recettes vient des déplacements interurbains alors que les coûts les plus importants sont en zone urbaine, en raison notamment des phénomènes de congestion qui y règnent.

\section{L'équité, la deuxième priorité}

Les préoccupations d'équité ne sont pas absentes du système des taxes sur les transports. Elles n'ont pas été étrangères à l'instauration de la TIPP, à une époque où l'automobile était un bien peu répandu réservé à une minorité de privilégiés. Mais c'est surtout au travers des subventions qu'elles s'expriment, comme le montre le tableau de l'encadré ci-contre. Elles ont pour but majeur de maintenir certains tarifs de transports en dessous des coûts et d'aider au financement des investissements. Cette pratique est courante dans les transports publics urbains et ruraux, pour lesquels le rapport entre tarif et coût varie entre un demi et un quart selon les agglomérations, ainsi que dans les transports ferroviaires régionaux. Elle est peu utilisée dans les transports ferroviaires interurbains et seules de très limitées catégories d'usagers (familles nombreuses, par exemple) en bénéficient. 


\section{les dépeNSES PUBLIQUeS DANS Les TRANSPORTS}

Les dépenses publiques dans les transports s'élèvent à 45 millions d'euros environ. Elles représentent soit des subventions aux opérateurs ou aux usagers, pour environ 11 milliards d'euros, soit des dépenses directes, pour environ 34 milliards d'euros. Les dépenses directes s'appliquent pour moitié à la gestion des infra- structures de voierie et pour moitié à l'exécution par les collectivités territoriales de services de transport de personnes (transports urbains et scolaires notamment). Le tableau cidessous en donne une décomposition par catégories de dépenses.

É.Q.

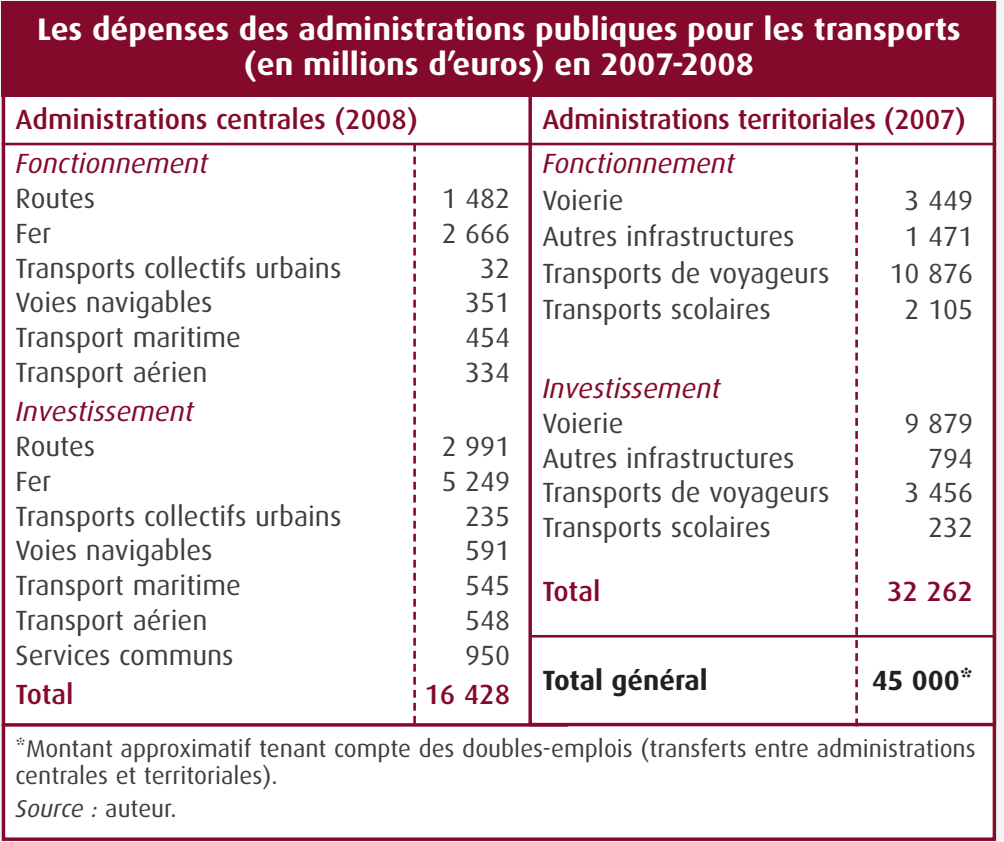

\section{L'orientation des choix, un objectif quasiment absent}

L'utilisation des instruments fiscaux dans un but d'efficacité, pour orienter les choix des usagers, est beaucoup plus rare. De toutes les taxes et subventions figurant dans les tableaux I et 2 , quatre seulement poursuivent ce dernier objectif.

La première est la taxe à l'essieu. Celle-ci a été instituée il y a environ 40 ans. D'un montant forfaitaire annuel qui dépend du poids des essieux du camion, elle avait pour but d'internaliser les dégâts occasionnés par les poids lourds aux chaussées ; mais cette taxe n'a guère été modifiée depuis son instauration et ne représente maintenant qu'une part limitée des dommages en cause. 
La taxe sur les nuisances sonores aériennes est assise sur le bruit émis par les avions lors de leur atterrissage ou décollage et son produit est utilisé pour insonoriser les logements riverains des aéroports. La subvention aux conteneurs est destinée à promouvoir le transport par conteneurs, plus respectueux de l'environnement que le transport routier. Enfin, l'éco-pastille a pour but de favoriser l'achat d'automobiles à émissions de $\mathrm{CO}_{2}$ réduites ; elle se matérialise par des bonus pour l'achat des automobiles économes en ce domaine et des malus pour l'achat d'automobile dépensières. Prises ensemble, ces quatre taxes et subventions ne s'élèvent qu'à 800 millions d'euros, un montant extraordinairement faible comparé à un total d'une cinquantaine de milliards de taxes et subventions.

Certes, dans la mesure où elles bénéficient essentiellement aux transports collectifs et où elles modifient le partage modal en faveur de modes moins agressifs pour l'environnement que la route, les subventions ont un effet sur les coûts externes, mais cet effet est assez limité car les reports modaux sont réduits.

Il en est de même pour la TIPP qui a deux effets sur la demande. D'abord, en renchérissant le mode routier, la TIPP influe sur les choix modaux et joue donc en faveur des modes collectifs, en général plus favorables à l'environnement. Mais cet effet est limité car l'élasticité des reports modaux est faible. En effet, dans beaucoup de situations, le choix du mode est contraint ; en outre il est aveugle, il incite uniformément au report modal, sans distinguer les situations où il est peu ou pas souhaitable de celles où il l'est extrêmement. Cet effet est aussi très global, il ne cible précisément ni la congestion, qui est certes un peu reliée à la consommation de carburant, mais qui varie dans des proportions considérablement plus fortes (voir le premier encadré de cet article), ni la pollution de l'air, ni le bruit. La TIPP agit aussi plus directement sur la consommation pétrolière et contribue à la réduire ${ }^{3}$. Mais cet effet n'a pas été voulu par le législateur ; on doit d'ailleurs remarquer que le taux de la TIPP est nettement plus élevé que l'externalité de l'énergie carbonée. Si en effet on retient comme coût collectif de la tonne de $\mathrm{CO}_{2}$ émise la valeur établie par le rapport du Centre d'analyse stratégique (CAS) ${ }^{4}$, la taxe frappant le litre de carburant devrait se situer à 0,06 euro par litre, une valeur bien inférieure au niveau actuel de la TIPP qui varie de 40 à 60 centimes par litre selon le type de carburant et la région ${ }^{5}$.

La différence dans l'accent mis sur les trois motifs d'utilisation des instruments fiscaux est frappante. L'essentiel de la tarification des transports a été conçu pour alimenter les caisses publiques. L'équité est un autre objectif d'importance significative. Mais l'efficacité, la recherche d'une orienta-

3. Ce sont les différences de taxation des carburants entre les États-Unis et l'Europe qu'on invoque pour expliquer les différences de consommation des automobiles des deux continents.

4. QUINET Alain (président de la commission en charge du rapport). La Valeur tutélaire du carbone. Paris : La documentation Française / CAS, 2009. À noter qu'il s'agit là d'une valeur actuelle qui devrait croître rapidement, au rythme de $3 \%$ par an.

5. On voit là que les débats sur la taxe carbone n'auraient pas dû concerner les transports routiers. 
tion adéquate des décisions des usagers, est presque totalement absente du système des taxes et subventions.

$\mathrm{Au}$ total, tout se passe comme si les autorités publiques trouvaient dans le secteur des transports, notamment la route, un domaine privilégié de ponction fiscale en raison de la capacité à payer de l'automobile. Indépendamment de cela, elles décident de façon discrétionnaire les dépenses dans les transports, essentiellement orientées vers la réalisation d'infrastructures ou des subventions à des activités soutenues essentiellement pour des raisons d'équité et de distribution. Il s'agit ainsi d'accompagner la demande, de la satisfaire, éventuellement de l'aider à s'exprimer par des subventions là où les coûts sont élevés, mais absolument pas de l'orienter dans le sens d'une plus grande efficacité collective. C'est une politique d'offre, pas une politique de demande.

\section{Des tarifs de transports ne visant pas l'efficacité}

On pourrait penser que les effets d'orientation des choix des usagers sont obtenus, non par la taxation publique, mais à travers les tarifs que les opérateurs de transport proposent à leur clientèle. Or il n'en est rien. On rencontre dans les transports deux sortes de tarifs, ceux payés pour l'usage des infrastructures là où elles sont payantes, et ceux payés pour les services de transport proprement dits. En ce qui concerne la première couche, celle de l'usage de l'infrastructure, sont concernés les aéroports, les charges que le gérant des infrastructures ferroviaires — Réseau ferré de France (RFF) fait payer aux exploitants ferroviaires (au premier rang desquels figure la SNCF, Société nationale des chemins de fer), les autoroutes à péage et le stationnement payant, la circulation sur les routes ordinaires étant gratuite. Le tableau 3 fournit pour chaque mode les recettes dégagées et les grands principes de fixation des charges unitaires correspondantes.

On voit que dans la détermination des charges d'infrastructures, il n'y a aucune prise en charge des externalités, sauf très partiellement pour le chemin de fer dont les charges sont modulées grossièrement en fonction des

\begin{tabular}{l}
$\begin{array}{c}\text { Tableau 3 - Les charges d'infrastructures : montants } \\
\text { (en millions d'euros) et modalités de fixation en } 2008\end{array}$ \\
\begin{tabular}{|l|c|l|l|}
\hline Mode concerné & Montant & \multicolumn{1}{|c|}{ Assiette de tarification } \\
\hline Chemin de fer & 2,7 & Somme d'un terme lié au train, d'un terme lié à la dis- \\
tance et d'un terme lié à la densité de trafic de la ligne \\
Voies navigables & 0,2 & Liée au tonnage et au kilométrage \\
Autoroutes & 7,1 & Liée au type de véhicule et à la distance parcourue \\
Ports maritimes & 0,7 & Liée au tonnage et au type de marchandise \\
Aéroports & 2,9 & Tarif par passager et péage lié au poids de l'avion \\
Stationnement public & 1,5 & Proportionnel à la durée de stationnement \\
Total recettes de péage & 15,1 & \\
\hline Source : auteur.
\end{tabular} \\
\hline
\end{tabular}


coûts d'infrastructure et de la densité du trafic, donc de la congestion. Les péages autoroutiers ont un effet négatif en termes d'orientation de la demande puisqu'ils frappent des voies dans l'ensemble moins congestionnées que les routes libres de péage. De même, dans l'aviation, les taxes ne sont pas modulées en fonction de l'heure et ne tiennent donc pas compte de la congestion aérienne.

En ce qui concerne les opérateurs de services, ceux qui assurent le transport de voyageurs ou de marchandises — c'est-à-dire les compagnies aériennes, les compagnies de transports régionaux et urbains et la SNCF les tarifs qu'ils proposent sont soit libres, soit fixés par la puissance publique. Là où les tarifs sont libres, c'est le jeu du marché qui prévaut. Les opérateurs fixent alors leurs tarifs de façon à maximiser leurs profits. Ils ne s'occupent pas des effets externes. Là où les tarifs sont encadrés par la puissance publique, c'est-à-dire essentiellement dans les transports urbains et régionaux, ils sont en général fixés à une valeur moyenne qui gomme les différences de situations et qui ne cible pas les effets externes; ils répondent plus à des soucis d'équité, d'ailleurs inscrits dans la loi fondamentale d'organisation des transports intérieurs (LOTI) ${ }^{6}$ qui précise que les transports sont un service public auquel chacun doit avoir accès dans des conditions raisonnables de production et de prix. Mais aucune trace d'une quelconque correction des externalités, à laquelle pourtant la LOTI incite puisqu'elle énonce également que les choix doivent tenir compte des « coûts économiques réels et des coûts sociaux dont ceux des atteintes à l'environnement ».

Ainsi la fonction d'orientation de la demande et de correction des externalités n'est pas plus remplie par la tarification mise en place par les opérateurs de transports qu'elle ne l'est par les taxes et subventions publiques.

\section{Les gains à attendre d'une bonne orientation de la demande}

Il y aurait pourtant amplement matière à corriger ces effets externes qui nuisent à l'efficacité économique. On a évalué dans plusieurs études les conséquences bénéfiques qu'aurait une telle internalisation, en termes de réduction des atteintes à l'environnement, de gain de temps, de diminution de la congestion et d'amélioration de la sécurité. Reprenons ici les résultats issus de l'étude européenne Revenue ${ }^{7}$. Cette étude internationale comportait un volet français dans lequel étaient distinguées la région parisienne, les autres zones urbaines et la rase campagne. Le tableau 4 en présente les principaux résultats.

6. Loi d'orientation des transports intérieurs, dite LOTI, n 82-II53 du 30 décembre I982. Cette loi constitue le cadre d'organisation du système des transports et définit les grands objectifs que doit suivre la politique menée par la puissance publique dans le secteur.

7. ROY Rana (sous la dir. de). Revenues from Efficient Pricing: Evidence from the Member States. Paris / Bruxelles : UIC (Union internationale des chemins de fers) / CER (Community of European Railway and Infrastructure Companies) / Commission européenne (DG-TREN), 2000. 
des différences encore plus marquées. Avec des taxes optimisées, les trafics routiers en zone urbaine seraient sensiblement réduits, de l'ordre de I5\%, alors que les trafics en rase campagne seraient à peu près inchangés. L'analyse des résultats pour les autres modes de transports, non figurés ici, ferait apparaître des accroissements de fréquentation liés à un transfert du mode routier vers les modes collectifs, aussi bien en zone urbaine qu'en rase campagne.

Si maintenant on examine les recettes globales tous modes confondus et tous types de véhicules, on constate que les recettes issues de la taxation optimisée augmenteraient : les pouvoirs publics recueilleraient davantage de fonds, mais la répartition selon les localisations serait très différente. Les recettes issues de la taxation des transports interurbains seraient à peu près inchangées, mais celles issues des taxes urbaines seraient sensiblement plus fortes. $\mathrm{Au}$ total, les recettes publiques augmenteraient de près de $30 \%$. Quant au bien-être collectif, il augmenterait d'un montant qui, dans les hypothèses de la simulation, est évalué à Io milliards d'euros, soit un ordre de grandeur de $20 \%$ du montant des taxes perçues; ce montant représente les gains en termes de temps, de sécurité et de coûts externes d'environnement permis par le caractère incitatif de la tarification. Et encore ne compte-t-on pas ici les gains dits de double dividende : les taxes spécifiques supplémentaires perçues par rapport à la situation actuelle permettraient, à recettes fiscales constantes, de réduire d'autant d'autres taxes générales qui ont un effet négatif sur le développement économique, par exemple les taxes frappant le travail et qui freinent l'emploi.

\section{Les obstacles ò une tarification incitative}

Pourquoi une structure de taxation comportant de si évidents bénéfices n'est elle pas mise en œuvre ? Il y a à cela de nombreuses raisons, certaines logiques et d'autres plus artificielles.

\section{L'équité}

On doit d'abord remarquer que, souvent, l'efficacité est en contradiction avec l'équité. Selon un exemple classique, une tarification de la congestion routière en zone urbaine, spécialement élevée aux heures de pointe, frapperait les flux domicile-travail de plus en plus composés de personnes à revenus moyens ou inférieurs au fur et à mesure que s'est étendue la possession d'automobile. On peut trouver au cas par cas des réponses à ce genre d'objection ; ainsi la tarification de la congestion à l'heure de pointe devient plus équitable si elle s'accompagne d'un développement de modes alternatifs publics suffisamment fréquents et bon marché, que l'on peut financer justement par les recettes de la taxe de congestion.

\section{L'acceptabilité}

Dans un ordre d'idées voisin, l'action sur les taxes rencontre une vive opposition de la part de ceux qui en pâtiraient ou des professionnels dont 
elle diminuerait la clientèle ; ces parties prenantes utilisent alors leur pouvoir d'intervention pour en neutraliser les effets. C'est pourquoi les réformes de taxation sont si rares. Ainsi, au niveau européen, alors que l'harmonisation des tarifs d'infrastructures est un but officiel de la Commission depuis le début de l'Europe en I958, les décisions en ce domaine ont été extrêmement limitées. Elles s'en tiennent à l'affirmation de grands principes comme la transparence et la non-discrimination. Mais dès qu'on s'aventure dans une direction qui pourrait conduire à imposer des montants précis, les discussions n'en finissent pas, les décisions pataugent.

Le premier pas, qui sera la directive Eurovignette, ayant pour but de fixer les règles de tarification des poids lourds pour l'usage des routes principales, est en discussion depuis de nombreuses années, sans que l'on soit encore totalement assuré du point d'aboutissement ni de sa date, même si maintenant elle semble proche. N'est-ce pas pourtant un des rôles essentiels des pouvoirs publics que de mettre l'intérêt général au-dessus des intérêts particuliers ?

\section{Le corporatisme}

Ces intérêts particuliers prennent, dans les transports, une forme particulière, celle du corporatisme. Pendant longtemps, le secteur a été organisé par filières modales, que l'on retrouvait au niveau tant de la profession que des syndicats et même de l'administration. Dans une telle situation, il n'est pas question d'influer sur la demande de chaque mode, il faut au contraire la satisfaire et pour cela trouver des ressources financières.

C'est cette orientation qui a conduit, dans le passé, à développer des politiques d'investissements dans des infrastructures indépendantes d'un mode à l'autre, et qui perdure avec l'idée qu'il suffirait d'investir dans les modes économes en environnement, essentiellement les transports collectifs, pour réduire sensiblement les atteintes en termes d'externalités. Il faut dissiper cette illusion. À elle seule, la construction d'infrastructures ne peut modifier profondément le partage modal. On l'a bien constaté dans les expériences de développement des transports collectifs urbains : l'augmentation de fréquentation que ces transports collectifs ont connue s'est produite pour une part importante du fait d'usagers nouveaux qui, auparavant, ne se déplaçaient pas ; une autre part, plutôt minoritaire, vient d'anciens usagers de l'automobile ; mais la place qu'ils ont libérée sur les routes et dans les rues a été occupée par de nouveaux automobilistes qui ont profité des conditions de circulation améliorées. Une autre illustration de cette incapacité de la politique d'infrastructure, prise isolément, à bien orienter les choix des usagers se lit dans le bilan des liaisons ferroviaires à grande vitesse en termes de trafic, dont les chiffres clefs sont indiqués dans le tableau 5 (page suivante), qui montre que l'effet du TGV (train à grande vitesse) sur la réduction du trafic routier est négligeable ; il ne correspond guère plus qu'à une ou deux années de croissance du trafic routier.

Ces exemples montrent que le seul développement des infrastructures d'un mode ne peut suffire, sauf justement à être accompagné d'une tari- 


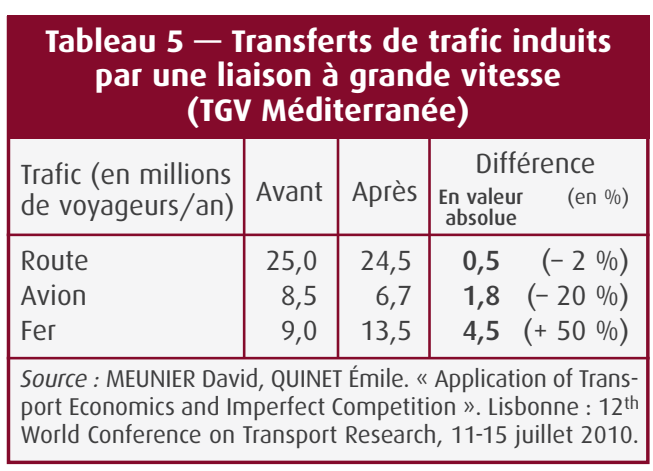

fication adéquate du mode concurrent. Ajoutons que cette politique de développement des infrastructures présente en outre l'inconvénient d'être dispendieuse pour les deniers publics, alors que l'action sur la demande et l'usage de taxes incitatives rapportent au lieu de coûter.

Certes, la situation évolue, mais lentement. Si beaucoup d'opérateurs de transport de marchandises jouent depuis longtemps la carte de l'intégration logistique, cette orientation n'est qu'assez récente à la SNCF. Quant aux opérateurs de transport de voyageurs, ils restent très largement unimodaux, au moins à l'intérieur de l'Hexagone ${ }^{8}$.

\section{Les découpages et règles administratives}

À ces raisons s'ajoutent des considérations organisationnelles. Les premières tiennent au découpage des autorités et aux contraintes législatives auxquelles elles sont soumises. Les taxes sont décidées par le Parlement et s'appliquent sur l'ensemble du territoire, alors que les collectivités territoriales n'ont à leur disposition que la tarification du stationnement public et, pour les ouvrages nouveaux, la possibilité d'y instaurer le péage. Il y a là un domaine que la décentralisation n’a pas touché, alors que précisément, comme le montre la variabilité des coûts externes selon la localisation, elle y aurait tout son sens.

En outre, les interactions entre collectivités sur le plan de ces coûts externes sont mal prises en compte par la répartition des pouvoirs de décision. Ainsi, la plupart des décisions concernant la circulation sont prises au niveau de la commune mais touchent l'ensemble de l'agglomération où se situe la commune ; c'est le cas du stationnement et de ses tarifs. Cette incohérence ne concerne pas seulement la tarification ; ainsi une commune qui crée une zone industrielle sur son territoire va en bénéficier sur le plan de ses recettes fiscales, mais cette zone va créer du trafic dans l'ensemble de l'agglomération, et donc des nuisances et de la congestion dans les communes voisines.

\section{Les coûts de transaction}

Une autre difficulté de la taxation incitative réside dans les coûts de transaction qu'elle peut entraîner, d'autant plus élevés que les effets externes à

8. Si par exemple Veolia ne gère que des autobus et autocars en France, cette entreprise gère aussi bien des trains que des bus ou même des aéroports à l'étranger. 
combattre sont complexes et variables selon les situations. Ainsi, la pollution émise par un véhicule dépend du type du véhicule, de son âge, de son état d'entretien et de l'intensité de l'usage qui en est fait. De même, le coût de congestion d'un véhicule varie considérablement selon le lieu et l'heure de son trajet. Cibler correctement et avec précision ces effets peut se révéler techniquement impossible et conduit souvent à préférer des moyens de nature réglementaire, moins raffinés mais plus simples dans leur mise en œuvre. C'est ainsi que l'essentiel des progrès considérables obtenus en matière de pollution automobile provient des normes de plus en plus sévères édictées pour les émissions des véhicules. Car les instruments disponibles jusqu'à maintenant pour mettre en ouvre la taxation étaient bien pauvres, surtout dans le mode où elle apparaît le plus souhaitable, la route.

De quels outils dispose-t-on ? Les plus traditionnels sont la taxation des carburants et des véhicules, les péages autoroutiers et les tarifs de stationnement. Or, la taxation des carburants ne cible que la consommation d'énergie. Les péages autoroutiers sont, du point de vue de la congestion, anti-incitatifs puisqu'ils frappent les itinéraires les plus fluides. On pourrait cependant imaginer que, à partir d'un niveau moyen fixé pour des raisons de couverture des dépenses, ces péages soient modulés selon l'heure, plus élevés à l'heure de pointe et plus bas aux heures creuses. Mais cette modulation ne se rencontre que sur un nombre très limité de sections d'autoroutes (AI à l'arrivée sur Paris, A86 dans sa section à péage entre Saint-Germain-en-Laye et Versailles, le tunnel Prado-Carénage à Marseille). Le stationnement payant vise à réduire le trafic automobile, mais étant en général proportionnel à la durée, il favorise les déplacements de courte durée, ce qui a tendance au contraire à augmenter le nombre de déplacements.

\section{Les changements en cours}

\section{Les nouveaux moyens technologiques}

Mais les choses sont en train de changer sur tous ces plans. Ainsi, au niveau des moyens techniques, on assiste à un élargissement des moyens de perception des taxes permettant une meilleure adaptation aux cibles que constituent les coûts externes. On a vu d'abord s'instaurer des dispositifs de vignettes dont l'acquisition est obligatoire pour circuler à l'intérieur d'une zone urbaine. Les pays asiatiques ont été précurseurs en ce domaine, à Singapour notamment dans les années I990. Plus près de nous, la ville de Londres a mis en place en 2003 un péage pour entrer dans le centre-ville, péage qui fut ensuite étendu à une zone plus large 9 . La nouveauté de ce système n'est pas d'ordre technologique, mais plutôt organisationnelle, à la fois pour le paiement de la taxe (qui passe par une panoplie de possibilités allant de l'achat

9. Sur ce point voir LEAPE Jonathan. « Le péage urbain londonien ». Futuribles, $n^{\circ}$ 337, janvier 2008, pp. 49-7I (NDLR). 
dans les bureaux de tabac au paiement par SMS) et pour son contrôle (repérage photographique des plaques d'immatriculation).

La vraie innovation technologique repose sur les nouvelles technologies de l'information et de la communication (NTIC). Elle est fondée sur une communication sans fil entre un émetteur lié à l'infrastructure et un transpondeur installé sur le véhicule. Deux versions existent. La première consiste en une liaison satellitaire qui permet de localiser le véhicule en tout point du territoire grâce à un système GPS (Global Positioning System) ; il est alors possible de moduler sa taxation aussi finement qu'on le souhaite selon son type, sa localisation et le moment. La seconde consiste en une liaison à courte distance (DSRC pour Dedicated Short-Range Communications) qui permet de localiser le passage du véhicule au droit d'un portique ; c'est le prolongement du système LiberT qui équipe déjà la plupart des autoroutes à péage françaises. Chacune des deux techniques a ses inconvénients et ses domaines de prédilection. Le système par GPS est d'un coût initial élevé, dont on peut espérer qu'il diminuera par effet d'apprentissage ; il est bien adapté pour des zones étendues au réseau complexe, comme c'est le cas d'un pays complet. Le système de détection local DSRC est d'un coût moindre, il convient là où il suffit de cerner le trafic en quelques endroits clefs, par exemple dans les zones urbanisées denses.

\section{La progressive disparition du corporatisme}

Le corporatisme va probablement voir sa disparition accélérée. Il était fondé sur la séparation des modes et sur l'existence de services publics cloisonnés. Ces cloisonnements sont en train de se réduire sur tous les plans. Au niveau administratif, celui de l'organisation du ministère des Transports, on a vu récemment disparaître les directions sectorielles, et en particulier la direction des routes, au profit d'une direction générale des transports à vocation intermodale. Au niveau des opérateurs, l'introduction de la concurrence dans les services se développe, elle va — c'est un de ses objectifs susciter des innovations dans les services offerts aux utilisateurs, et l'une de celles que l'on peut escompter, c'est le développement de l'intermodalité.

Celle-ci s'est observée dans les pays étrangers qui sont en avance sur la France dans cette évolution. La SNCF s'oriente nettement dans cette direction pour les transports de marchandises : par sa filiale Geodis, dont la taille est supérieure à celle de la branche fret de l'entreprise mère, elle s'échappe du carcan modal et devient un opérateur multimodal de dimension mondiale ; elle ne fait en cela qu'imiter la Deutsche Bahn, engagée dans cette voie depuis déjà plusieurs années. Il est à prévoir qu'il en ira de même pour les voyageurs ; la concurrence est déjà possible sur les liaisons internationales et devrait d'ici quelques années l'être aussi sur les liaisons intérieures. Lorsqu'il en sera ainsi et que les régions pourront choisir leur opérateurs pour les services à courte distance, sans être obligées de recourir à la SNCF, on verra se diversifier l'offre, des services routiers se substituer aux services ferroviaires ou leur devenir complémentaires, alors qu'actuellement, les deux activités sont largement séparées. 


\section{L'émergence d'une acceptabilité politique}

Une autre évolution se fait jour, celle des mentalités et de la disposition de l'opinion à accepter ce type de tarification en Europe. Le mouvement a été imprimé par l'expérience londonienne de congestion charge ${ }^{\text {IO }}$ qui, au départ, a été jugée très risquée sur le plan politique ; mais l'audace du maire de l'époque a été couronnée de succès, le péage a été plébiscité.

Du coup, on a vu le péage apparaître en de multiples endroits. À l'échelle d'un pays, il était déjà en fonction en Suisse, depuis 200I, pour les poids lourds dont il cible les atteintes à l'environnement: chacun d'eux est équipé d'un compteur de distance parcourue, qui est relevé tous les ans, et la taxe payée est alors le produit de la distance relevée sur ce compteur par un tarif unitaire qui dépend du type du véhicule et varie avec son standard de pollution. Sous forme électronique, le péage existe aussi depuis plusieurs années en Allemagne pour les poids lourds, où il cible l'environnement et les dégâts causés aux routes ; il a également été installé en Autriche et en Slovaquie, et en milieu urbain à Stockholm, Oslo et Trondheim, ou encore Milan. Par ailleurs, l'Union européenne pousse le projet de directive d'Eurovignette qui aura pour but de faire payer aux transports routiers de marchandises leurs coûts externes, et l'on peut penser que les longues discussions auxquelles ce projet a donné lieu vont finir par aboutir dans des délais pas trop éloignés.

En France même, les choses bougent, et cela est largement dû au mouvement créé par le Grenelle de l'environnement ${ }^{\text {II }}$. Pour la première fois depuis longtemps, on a parlé de taxe sur le carbone ${ }^{\mathrm{I} 2}$, une taxe purement incitative, même si le projet a finalement été abandonné, et même si, comme on l'a vu, ce projet n'était pas bien adapté au cas des transports. Par ailleurs, pour se conformer aux directives européennes existantes et à venir, la France va mettre en place un système d'éco-taxe qui frappera la circulation des poids lourds ; ce système d'éco-taxe sera payé sur les routes principales et autoroutes libres de péage ; les décisions finales n'ont pas encore été prises, mais les informations disponibles donnent à penser que c'est un système par GPS, donc susceptible de couvrir tout le territoire, qui sera probablement choisi. Enfin, la loi dite «Grenelle II » fait sauter le verrou juridique qui empêchait la mise en œuvre généralisée du péage : elle autorise les agglomérations à percevoir un péage qui ne soit pas simplement la couverture des coûts de construction d'une voie nouvelle ; on peut donc désormais installer un péage sur les voies existantes, celles qui sont le plus congestionnées.

Io. Voir LEAPE Jonathan. Op. cit.

II. Processus participatif lancé en 2007 en France, visant à prendre des décisions de long terme en matière d'environnement et de développement durable, ayant notamment débouché sur deux séries de lois (Grenelle I en juin 2009, et Grenelle II en juillet 20I0) (NDLR).

I2. Sur ce point, voir notamment PERTHUIS Christian (de). «Quel avenir pour la taxe carbone en France? Les choix économiques après la censure du Conseil constitutionnel ». Futuribles, $\mathrm{n}^{\circ}$ 361, mars 2010, pp. 5-I4 (NDLR). 


\section{L'apparition d'une industrie nouvelle}

La conjonction de l'acceptabilité politique croissante et des possibilités nouvelles offertes par la technologie va entraîner l'extension d'une activité nouvelle dont on voit les prémices et que l'on pourrait appeler l'« infostructure », par opposition aux deux couches classiquement distinguées dans les transports : les infrastructures où l'on gère des ouvrages de génie civil, et l'exploitation des services où l'on gère des véhicules. Cette couche se distingue des deux précédentes à la fois par ce qu'elle produit et par les techniques de base qu'elle utilise. Ce qu'elle produit, ce sont tous les services immatériels d'information, de tarification, de repérage et d'appariement, qui prennent une importance croissante dans la gestion et l'organisation. Quant aux techniques qu'elle utilise, ce sont essentiellement les NTIC.

L'infostructure pointe déjà dans de nombreux domaines ; ainsi la tarification et l'information des usagers du transport aérien, comme de plus en plus celle des voyageurs ferroviaires, est fournie par des systèmes informatisés de réservation (SIR) qui ont été mis en place par des groupements de compagnies ; on a vu comment elle pouvait se déployer pour la perception de taxes et tarifs permettant de cibler exactement les coûts du transport. En matière de transport de marchandises, la logistique, qui gère les mouvements de matières dans et entre les entreprises, met en jeu certes les transports et ses deux composantes de base, les infrastructures et les véhicules, mais elle utilise aussi de façon beaucoup plus stratégique, pour la gestion des stocks et les approvisionnements « juste à temps », l'informatique et les NTIC sous toutes leurs formes.

Ce sont les mêmes outils de communication à distance entre des opérateurs et les véhicules qui permettent de développer l'information des usagers. On connaît déjà l'utilisation des systèmes GPS pour renseigner les automobilistes sur l'itinéraire le mieux adapté ; les plus sophistiqués d'entre eux prennent en compte l'état actuel du trafic. Les principaux opérateurs de transports publics mettent à la disposition de la clientèle des informations sur la fréquence et les temps de trajet. Toutes ces informations utilisent des systèmes de repérage des véhicules analogues à ceux que le péage peut utiliser. Au-delà de ces innovations en cours de diffusion, on peut imaginer la multiplicité des possibilités ainsi offertes pour la gestion de la demande et des flux de trafic, par exemple le développement de l'information permettant à chaque usager potentiel de gérer la combinaison des modes de transports qu'il utilisera, et l'importance accrue de la gestion des centres d'échanges, telles les gares ferroviaires qui deviendront de plus en plus, non pas seulement des endroits aménagés pour monter dans les trains et en descendre, mais des lieux d'information, d'échanges multimodaux et de coordination des activités qui peuvent s'y concentrer : le stationnement, la coordination avec les transports urbains, les services à la clientèle (commerces...). Dans une vue futuriste, on peut également envisager dans les agglomérations des systèmes d'enchères par lesquelles on achèterait par Internet ou par téléphone, sur un marché de droits, une vignette nécessaire pour circuler le lendemain ou un jour ultérieur dans une tranche horaire donnée. On peut également 
penser à la gestion par des moyens analogues des places de parking, en combinant la tarification des parkings et la généralisation de l'information fournie à l'automobiliste sur les emplacements disponibles aux alentours de l'endroit où il cherche à stationner.

Les techniques qui vont ainsi prendre une importance croissante sont très différentes de celles qui prévalaient jusqu'ici dans les transports. Elles s'appuient sur l'informatique et les télécommunications, alors que les techniques les plus fréquentes dans le secteur sont le génie civil pour les infrastructures et la mécanique pour l'exploitation des services. Ces NTIC et leurs applications au secteur des transports peuvent donner lieu à des mutations difficilement prévisibles, à l'inverse du génie civil et de la mécanique, des disciplines mûres et en progrès lent.

De ce fait, la taxation ne sera plus seulement un appendice de la gestion des budgets publics ou un complément au fonctionnement des infrastructures. Soutenue par une industrie propre et un métier dynamique en émergence, elle disposera de forces de développement d'autant plus efficaces qu'elle entre en synergie avec les autres pans de l'infostructure, l'information des usagers, la coordination.

\section{Des problèmes de gouvernance}

Le développement de cette nouvelle couche n'ira pas sans poser des problèmes de gouvernance. Citons-en deux, parmi les plus évidents. Le premier concerne le financement et la gestion des infrastructures, et notamment des infrastructures routières. La tradition française est fondée sur les autoroutes à péage, dans lesquelles une société concessionnaire, généralement émanation d'un groupement d'entreprises de travaux publics, réalise l'infrastructure et se paie sur le produit des péages qu'elle perçoit tout au long de la durée de vie de la concession. On connaît les difficultés et inconvénients qu'entraîne ce système en termes de régulation de l'entreprise concessionnaire, de sa gestion de l'entretien et de la fixation du niveau de péages qu'elle est autorisée à percevoir. Ce système est maintenant concurrencé par un autre système, qui se met en place en Allemagne par exemple, dans lequel la construction de l'infrastructure est dissociée de la perception des péages : la construction est réalisée sous la forme d'un marché de travaux publics classique ; l'entretien peut faire l'objet de contrats à durée limitée renouvelables ; quant à la perception des péages, elle est sous-traitée à une société dont le métier essentiel est l'informatique. Ce dernier système a pour avantages une régulation plus aisée et plus souple, et la réduction de la prime actuellement prise par le concessionnaire pour s'assurer contre le risque de trafic, le principal risque des concessions.

Le second problème de gouvernance que le développement de l'infostructure devrait entraîner concerne la propriété de l'information. Ce problème est déjà apparu dans le cas des SIR (systèmes informatisés de réservation) du transport aérien : les systèmes en cause étaient naturellement orientés de façon à faire apparaître en premier les conditions d'horaires et 
de prix des compagnies les plus influentes dans le groupement, et laissaient complètement sous silence tout ce qui concernait les compagnies hors groupement. L'Union européenne a dû légiférer pour corriger ces tendances.

On voit poindre le même type de problème, en France, pour la gestion des gares de chemin de fer. Jusqu'à maintenant elles faisaient partie du monopole de la SNCF. Maintenant que ce monopole disparaît, elles doivent se préparer à accueillir et à fournir la même qualité d'information aux concurrents à venir ; la SNCF d'ailleurs se prépare à toutes ces évolutions qui feront des gares et de leur gestion un service public à part entière, séparé de celui de l'exploitation des trains de l'entreprise. Des problèmes analogues de disposition des informations vont certainement apparaître quand il s'agira de partager des informations multimodales ou d'utiliser à des fins de tarification des données élaborées par des entreprises privées à des fins d'information de leurs abonnés. Les choix sont classiques dans ces situations d'informations : obliger à une diffusion complète de l'information est bon sur le court terme, car l'information est un bien public ; mais sur le long terme, une telle diffusion découragera l'incitation à créer cette information.

On se trouve donc à un moment charnière pour la taxation des transports, et plus généralement pour leur gestion. On était jusqu'à maintenant, et on est encore, dans une phase où les instruments financiers et fiscaux publics étaient essentiellement orientés vers la fourniture de ressources financières pour l'adaptation de l'offre à la demande, sans chercher à influer sur cette demande. L'assiette des taxes actuelles répond essentiellement à des critères d'acceptabilité par le public et d'équité ; leur destination est fondamentalement d'alimenter les caisses publiques ; cela a permis de développer une politique d'offre grâce à laquelle la France est dotée d'un système de transport abondant et de bonne qualité.

En revanche, les intentions d'orientation de la demande pour lutter contre les externalités jouent un rôle tout à fait mineur. Il y a à cela plusieurs raisons : d'abord les imperfections des moyens techniques pour cibler les effets externes ; ensuite la réticence des parties prenantes à toute réforme des taxes et tarifs, qui provoquent toujours des réactions négatives de la part de ceux qui en pâtissent, sans obtenir le soutien de ceux qui en bénéficient ; enfin, l'organisation des pouvoirs, très morcelés en matière de taxation, n'est pas favorable à une action cohérente. Ceci occasionne une perte d'efficacité importante ; les atteintes à l'environnement et la congestion ne sont pas réduites, les usagers ne sont pas incités à utiliser les moyens de transport les plus efficaces pour la collectivité.

Mais on peut penser que cette situation va changer sous l'influence de plusieurs facteurs. D'abord, certains mouvements de l'opinion commencent à se faire jour en faveur d'une taxation incitative ; une impulsion relayée par l'Union européenne et sa politique en faveur de l'environnement, et favorisée, 
en France, par le climat né du Grenelle de l'environnement. Surtout, le développement des NTIC offre des possibilités inédites de sophistication à la fois dans l'information aux usagers et dans le ciblage des effets externes. Enfin, la taxation des transports va se trouver partie prenante de l'infostructure, cette nouvelle couche qui est en train de se développer, à côté de la gestion des infrastructures et de celle des services. Cela pourrait donner lieu à des mutations importantes et difficilement prévisibles dans l'orientation du secteur, et permettre de passer d'une stratégie de développement de l'offre à une politique de gestion de la demande rendue d'autant plus nécessaire que les budgets publics se resserrent - tout ceci sous réserve que cette évolution soit surveillée pour éviter des erreurs dans la gouvernance des activités correspondantes.

\section{3e rencontre Jean-Jacques Salomon L'EMBRYON HUMAIN : SCIENCE ET DROIT \\ Jeudi 3 février 2011, 14h-18h30 - Futuribles International (Paris)}

\section{Intervenants :}

Florence Bellivier (professeur de droit à l'université de Paris X-Nanterre, spécialiste du droit civil, du droit de la bioéthique et du droit médical)

Valérie Gateau (philosophe)

Arnaud de Guerra (responsable de l'unité Recherches-projets européens à l'Agence de biomédecine)

Jacqueline Mandelbaum (membre du Conseil consultatif national d'éthique et responsable du service Biologie de la reproduction à I'hôpital Tenon)

Agnès Ricroch (généticienne et membre du comité de pilotage)

\section{Comité de pilotage :}

Julie Bouchard (enseignant-chercheur, université de Paris VIII-IUT de Montreuil ; chercheur associé au laboratoire Communication et politique, CNRS)

Pierre Hassner (directeur de recherche émérite du CERI)

Hugues de Jouvenel (directeur général du groupe Futuribles)

André Lebeau (professeur honoraire, CNAM)

Agnès Ricroch (maître de conférences à AgroParisTech, chercheur associé au laboratoire Écologie, systématique et évolution, université Paris Sud-CNRSAgroParisTech)

Claire Salomon-Bayet (professeur émérite de l'université Paris I)

Geneviève Schméder (professeur des universités, CNAM)

Inscription dans la limite des places disponibles (e-mail : anlebeau@wanadoo.fr) 


\section{CALENDRIER DES FORMATIONS VEILLE • PROSPECTIVE • STRATÉGIE}

FUTURIBLES INTERNATIONAL

- Introduction à la veille et à la démarche prospective

Animée par Hugues de Jouvenel, directeur général du groupe Futuribles, consultant international en prospective et stratégie

Jeudi 20 janvier 2011 - Prix : 956,80 euros TTC*

\section{- Méthodes et outils de la prospective stratégique}

Animée par Nathalie Bassaler, conseillère scientifique à Futuribles et viceprésidente de Prospective-Foresight, François Bourse, directeur d'études au GERPA (Groupe d'études ressources prospective aménagement) et enseignant-chercheur au LIPSOR (Laboratoire d'innovation, de prospective stratégique et d'organisation, CNAM), Hugues de Jouvenel, directeur général du groupe Futuribles, consultant international en prospective et stratégie, et Jacques Lesourne, président de Futuribles International Jeudi 27 et vendredi 28 janvier 2011 - Prix : 1435,20 euros TTC*

- Atelier de construction de scénarios contrastés Formation-action appliquée à la construction de scénarios contrastés

Animée par Véronique Lamblin, directrice d'études du groupe Futuribles, et François de Jouvenel, directeur d'études du groupe Futuribles

Mardi 8 février 2011 et mercredi 9 février matin - Prix : 1 136,20 euros TTC*

À photocopier et à retourner à Futuribles International, 47 rue de Babylone - F-75007 Paris Tél. 33 (0)1 53633771 - Fax 33 (0)1 42226554

croels@futuribles.com - www.futuribles.com

$\square$ Je souhaite recevoir gratuitement le programme des formations

$\square$ Je m'inscris au(x) formation(s)

Nom - Prénom

Fonction - Organisation

Adresse

Code postal .

Tél.

Ville/pays

Fax

E-mail

Règlement : $\square$ Chèque à l'ordre de Futuribles International

$\square$ Au reçu d'une facture

Remise de $10 \%$ pour inscription multiple dès la deuxième participation; dispense des frais de participation pour les membres partenaires de Futuribles International et remise de 50 \% pour les membres associés (valable pour une personne par session de formation). 